\title{
Research on the Functions of Users' Emotions in Social Media Product Design
}

\author{
Kaiyue Chen ${ }^{1}$ \\ ${ }^{1}$ Quanzhou No.5 High School Quanzhou, China
}

\begin{abstract}
With the prevalence of social media, which integrates into individuals' routine life, the emotions of users partially direct the content that published on platforms, and it has become an unignorable element during social networking sites operation. Appropriately utilizing emotions is an feasible and effective method of enhancing profit. To figure out the impacts that generated by the designs of social media platforms on users' emotions, and the corresponding influences of emotions on advertisements, this paper focuses on the designs, including the homepage and hashtags, and their functions on mainstream social networking services (SNS) inside and outside the domain, such as Sina Weibo, Twitter, Instagram, and TikTok. The effects of group users against individual users concerning emotion are discussed, and the positive and negative effects on devised advertisements when taking advantage of users' emotions are also analyzed.
\end{abstract}

\section{Introduction}

According to the report of USA Today published on April 22, users of Facebook could change the mode of a static heart-shaped emoji to a vibrating one. This was because under the severe epidemic situation of COVID-19, individuals encountering agony, solitude, quarantine, death, etc. urgently needed more emotional connections provided by social media, meaning that the significance of emotion should be paid more attention to. The questions of whether the designs of social media can affect the emotions of users and lead the generation of diverse feelings and which impacts will be exerted on advertisement arise.

On various types of social media platforms, individuals can express their emotions appropriately. With the continuous advancement of communicating, the transactional model contributes to the emotion transmission, increases connections, and blurs the disparity of network and real life, in terms of communication. Additionally, the eight key changes of the ways people communicate, which was influenced by social media, include anonymity, the richness and diversity of information, omnipresence, speed, the multitude of roles that users assume and their relationships to each other, the move from objectivity to subjectivity, the ability to combine information, and the near absence of traditional methods of regulation[1]. All of them are beneficial to the expression of emotion, enhancing the willingness of sharing emotions on social media. However, the model and findings can't effectively figure out and illustrate the issues concerning emotion; so the purpose of this paper is to address the questions mentioned above.

\section{The Designs of Mainstream Social Networking Sites Inside and Outside the Domains}

Social media consists of microblogs, photo-sharing platforms, video-sharing platforms, social networks, etc, each of which has disparate functions on bringing convenience for users, and satisfying various sorts of demands of individuals. People utilize social media organisms, which provide connectivity, to enrich their daily life, like communicating with friends online and offline, expressing their perspectives and thoughts, sharing and recording their life, connecting with celebrities and influencers, purchasing products, and so on.

\subsection{Homepage}

It's common to see the design of the homepage in the outside and inside domains of social media. Within the homepage, users can browse the contents published by the people they follow and the recommended ones in light of their interests.

On Twitter, the tweets posted by the followed users are presented chronologically; so are the contents they like. Popularity is shown under each tweet by presenting the numbers of comment, retweet, like and share. In addition, the comments created by the individuals whom users also follow are also shown. Users can choose whether the popular tweets or the latest ones will be presented on their homepages, and use a shortcut to post their tweets.

On Weibo[2], the homepage is similar to that of Twitter, but the comments and the favoured contents of people whom users also follow don't display. And if a user 
follows an e-community, the updated contents posted there will be revealed on the homepage as well. Users can choose to browse the contents published by different categories of the followed people, or the recommended Weibo merely. What promotes diversified is that users can add icons after usernames and can also adorn their profile photos, which is entertaining and amusing. The reposted content of different users are simultaneously present with the original Weibo, which differs from that of Twitter .

Twitter is relatively compact, convenient, and efficient. In the same period of time, Twitter users can browse more information, compared with Weibo. Users can represent emotions via their tweets, and this can also provoke an emotional response. [3]When skimming through different tweets, users, who are easily to be impacted, will change their feelings rapidly. In contrast, Weibo is fancy, attracting more young people than people of other age ranges. The ornament enables users to optimize their profiles and to feel the social enjoyment. Even though users can barely browse few Weibo in one interface, they are better participated in, since they can see a variety of comments concerning one content in the same time. These comments evoke the users' emotions, which depends on the contents that they skim. What they feel and consider are partially based on others' opinions.

\subsection{Hashtag}

By utilizing hashtag, one of the most efficient methods of dissemination, users can find the specific contents that they are interested in within a relatively short time. To improve user experience, many social networks adopt the function of tag. Hashtags online perform a range of linguistic[4] and pragmatic[5] functions, alongside their categorizing and searching functionalities.[6]

Instagram[7] allows users to add any tags when posting videos or photos. Through this way, more users can see the published contents under a particular topic and then, interact with each other. This method also makes it possible to increase users' participation while using Instagram, for the reason that users can gain a sense of satisfactory when they are noticed by others during communication. Additionally, influencers often use hashtags to exhibit their purposes when advertising. Due to Instagram's characteristic of focusing on photo sharing and its layout that the picture occupies most area, the effect of tags correspondingly recedes.

The function of hashtags on Twitter is virtually the same as that on Instagram. What different is that the tags on Twitter which are increasing in popularity will appear in the list of trend since they are frequently used by users. In this circumstance, users can know major events, latest politics, popular influencers, etc. Such substantial attention can create numerous profits.

TikTok[8] also enables users to append hashtags, so that users can watch videos concerning their desired topics. On TikTok, tag is extremely significant because the platform is assisted by algorithm, which gains users' preferences, therefore it will recommend the videos that users are probably interested in, according to the tags included in videos that users like. Even though they do not search the hashtags when watching the videos, these tags will be shown spontaneously in the videos that users are fascinated about, on the homepages.

Hashtags can inspire various emotional reactions of users, since individuals have disparate understanding regarding similar contents. Usually, different emotions may be expressed by users towards different topics. [9]As video variety continues to become more diverse, users find it increasingly more difficult to define video categories.[10] Employing hashtags concerning emotion meets the needs of seeking out targeted videos, and fulfills emotional support.

\section{The Guidance of Designs of Social Networks to Users' Emotions}

Emotion is a significant element when people surf the Internet. Users use social media platforms to intuitively satisfy their needs, like relaxing, entertaining, receiving information, communicating, etc. Appropriately utilizing emotion is a feasible and resultful method of gaining revenue.

\subsection{The Impact of Design of Social Media Products on Users' Emotions}

The designs of social media platforms leverage users' emotions, regarding to the layout of the homepage, the design of hashtags, the setting of search box, and so on. A design that seeks to evoke emotions should be wellfounded rather than subjective or intuitive.[11] To optimize using experiences, and to increase participation, designers seek effective methods of leading emotions.

\subsubsection{Layout of the Homepage}

The homepage includes various components, such as likes, comments, colors, separating lines, shortcut keys, and ratio of the layout of posted content, each of which is closely concerned with the fluctuation of emotion. The visibility of likes and comments benefits the increase of participation, enabling users to engage in interacting and communicating more deeply. The indispensability can be illustrated by a rule. In June, 2020, Sina Weibo launched a temporary restriction, which rendered widespread dissatisfaction: only users who follow a publisher for at least 7 days can comment. This measure partially weakened users' enthusiasms of using the platform, since the entry of expressing emotion was close, and netizens could not voice their opinions. Of the volunteers who were investigated, $32.53 \%$ claimed that they felt angry and upset, $32.54 \%$ deemed that they didn't have emotional changes, and approximately $21.43 \%$ were delightful during the period of restriction. 


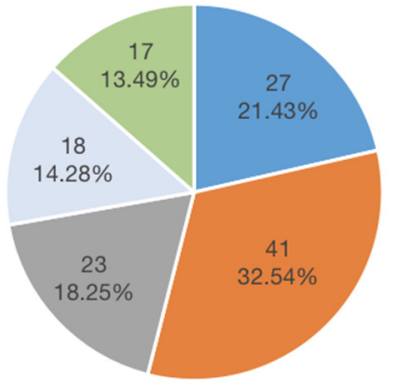

- delightful $\|$ unaffected $\mathbf{m}$ angry $=$ upset $=$ others

Fig 1. Users' feelings during the period of restriction

Additionally, the form of notification inspires different emotions. The red dots used on Facebook or the pink circles employed by Instagram to signify new messages or alerts appeal to a person's motivation to complete these tasks, and also their emotional need to see the newest contents from their social networks.[12] Pink circles on Instagram, which appear after people finish browsing all the contents posted by the followed users within one day, enables users to feel contented. Moreover, individuals have different preferences. The occupied area of each separating line is related with users' intuitive feelings. Wider line makes the interface more clear, enabling users to focus on every published content, since the number of incorporated content is less, while narrow line makes it possible for users to sense the feelings of different people simultaneously, knowing general sentiment.

\subsubsection{The Design of Hashtag}

Most social networks provides default categories for uploaders to use tags, so that users can perform keyword searches or to find content, according to their interests. [13]However, users sometimes have a hard time utilizing the basic categories of hashtags to precisely find content. Definitions of some contents are vague and unclear, which are difficult for users to distinguish. Adding tags about emotions is a practical method of dealing with such issue. As for some sentiments, users can not have consentaneous feelings as the presented hashtags, since the emotions are related to factors, like real-life conditions and experiences, personal understandings, and different objects that are specifically focused on. According to the result of an experiment conducted by Bianchi et al., designers can use the tags that are associated with happiness, sadness and fear, because the felt emotion matched with the tagged emotion. However, with regards of anger and disgust, it is not advisable for designers to use them as tagged emotions.[14] Utilizing suitable hashtags can gather more consistent feelings, enabling users to accurately understand the meanings and feelings of publishers rather than infer individual thoughts.

\subsection{The Impact of Group Users against Individual Users}

When using social networking sites, it seems that users' emotions are likely to be influenced by others in a specific environment where the majority of individuals have similar sentiments or opinions. For instance, people are likely to feel delighted when seeing a group of individuals share the process of celebrating an ceremony on social media platforms. However, the joviality is directed at the event, the ceremony itself, rather than a created certain emotion. The delight is an imagination that is irrelevant to the emotions of group users. According to an observation conducted by Debashis Naskar et al, the emotion state of a significant number of posts does not agree with the dominant emotion, indicating that influential ability of social propaganda on the social network is questionable.[15] X. Sun et al also authenticated that the emotion of micro-blogs released by group is subject to power-law distribution, while the individual user is not.[16]

\section{The Impact Created by Users' Emotions on Advertisements of Social Media}

Advertisement is an indispensable component when operating social networking, since advertising is a critical source of revenue for social media platforms - exceeding \$9bn in the second quarter of 2017 for Facebook alone[17]. Based on this, social media platforms continuously update and optimize their algorithms, and the models of advertising, and marketers seek ingenious methods of designing and presenting ads to maximize sales as well.

\subsection{The Impact Generated by Users' Emotions on Designs of Advertisements}

To effectively increase the viewership and availability of ads, emotion, undeniably, helps to complete the target. Personalized advertisements utilized datafication and big data, which are recommended to users according to their feelings, sentiments, likes, etc, to observably attract more users' attention. The gathered emotions affect the ads that users receive. Poels and Dewitte found that creativity leads to the pleasure and arousal reactions in consumers.[18] Because users' reactions, like enjoyable, surprising, etc, promote the clicks of advertisements creating numerous profit, marketers prefer to design creative and fancy ads. For users who have negative moods, like depression, frustration, indignation, the designs of ads would add and stress words, like "decompression", "diverting attention", "alleviating anxiety" etc. Setyani et al. concluded that the higher the perceived creativity was, the higher the users' hedonic motivation to click through the ad in search of novelty, variety and surprise would be. [19]Therefore, designs of advertisements and users' emotions interact with each other.

However, with the prevalence of applying emotions, some unscrupulous merchants, who exaggerate products' utilities and create false advertisements, utilize users' feelings to make illegal profits. Taking a victim of a dishonest trader on TikTok as an example;[20]one victim purchased a seemingly incredible useful product after watching a video that exhibited its utility. However, when he received no feedback and planned to refund the product, the merchant had been vanished. 
The marketer utilized consumers' curiosity and surprises to induce them to purchase the products. The emotions expressed by users are beneficial to the designs of advertisements, since the ads can be more diverse and fascinating, which enables platforms and marketers to obtain profit more efficiently. Nonetheless, the immoral behavior of taking advantage of users' emotions will consume people's trust and eventually make people lose faith toward platforms. The ongoing and lengthy process of constructing belief among platforms, merchants, and users will be ruined, despite elaborate and fined algorithms, artificial intelligence, big data, and datafication are applied. Over or improper utilizing emotions will render them feel offensive, which decreases the effectiveness of advertising.

\section{Conclusion}

Emotion gradually becomes an indispensable ingredient, which is supposed to be prioritized. With the continuing advancement of social media, some platforms ceaselessly optimize the designs to meet the demands of users' emotions under various conditions. This paper mainly analyzes the effect of users' emotions, which exerts on social media product design. The homepage of Twitter is compact and efficient, while that of Weibo is fancy. Hashtags on Instagram are relatively less significant, compared with those of Twitter and TikTok, which distinguishes the diverse effect that functions on users' emotions. The visibility and access of likes and comments on the homepage influence users' preferences of using the social media, and the symbols as well as the widths of separating lines potentially affect users' emotions. Categorizing hashtags by emotions makes it convenient for users to find the contents they desire to see, excluding feelings like anger and disgust. As for the impacts of group users against individual users, there are no necessarily relations. Empathy is perhaps an imagination towards a certain event. Personalized and creative advertisements undeniably are more likely to be accepted by users, so that the sales of products can be augmented to the expected and satisfactory amount in a relatively short time. However, inappropriately employing users' emotions will arouse the resistance of users, consume their beliefs, and render the efficiency of marketing to descend, even though the platforms are assisted by algorithm, datafication, big data, and artificial intelligence.

\section{References}

1. The New Power of Political Influence, CES (Center for European Studies) and Suomen Toivo Think Tank

2. A Chinese microblogging website that was launched by Sina Corporation. It's one of the biggest social media platforms in China. See: https://en.m.wikipedia.org/wiki/Sina_Weibo

3. Constance Duncombe. "The Politics of Twitter: Emotions and the Power of Social Media." International Political Sociology (2019) 13, 409-429

4. Zappavigna, M., 2015. Searchable talk: the linguistic function of hashtags. Soc. Semiot. 25 (3), 274-291.
5. Scott, K., 2015. The pragmatics of hashtags: inference and conversational style on Twitter. J. Pragmat. 81, 8-20.

6. Kate Scott, 2017. "Hashtags work everywhere": The pragmatic functions of spoken hashtags.

7. An American photo and video-sharing social networking service owned by Facebook, Inc. See: https://en.m.wikipedia.org/wiki/Instagram

8. A video-sharing social networking service owned by ByteDance, a Chinese company founded in 2012 by Zhang Yiming. See: https://en.m.wikipedia.org/wiki/TikTok

9. Jinpeng Chen, Yu Liu, Ming Zou, 2017. "User emotion for modeling retweeting behaviors." Neural Networks 96(2017)11-21

10. Yen-Liang Chen, Chia-Ling Chang, Chin-Sheng Yeh, 2017. "Emotion classification of YouTube videos"Decision Support Systems 101((2017)40-50

11. Boulton M (2009) Designing for the Web. Penarth: Mark Boulton Design Ltd Silveira LM (2011) Introduction to color theory (in Portuguese). Ed. UTFPR

12. Krishnan Vasudevan. Design of Communication: Two Contexts for Understanding How Design Shapes Digital Media. Journalism \& Mass Communication Quarterly 1-16. (C) 2020 AEJMC, cited in Eyal, N. (2014). Hooked: How to build habit-forming products. Penguin.

13. Yen-Liang Chen, Chia-Ling Chang, Chin-Sheng Yeh, 2017. "Emotion classification of YouTube videos" Decision Support Systems 101((2017)40-50

14. Renata G. Bianchi \& Vânia P. A. Neris \& Anderson L. Ara, Tags vs. observers - a study on emotions tagged and emotions felt with Flickr pictures, Multimedia Tools and Applications. Berlin: Springer Science+Business Media, LLC, 2019.

15. Debashis Naskar, Sanasam Ranbir Singh, Durgesh Kumar, Sukumar Nandi, and Eva Onaindia de la Rivaherrera. 2020. Emotion Dynamics of Public Opinions on Twitter. ACM Trans. Inf. Syst. 38, 2, Article 18 (March 2020), 24 pages.

16. X. Sun, et al., Detecting users' anomalous emotion using social media for business intelligence, $\mathrm{J}$. Comput. Sci. (2017), http://dx.doi.org/10.1016/j.jocs.2017.05.029

17. Cherniece J. Plume \& Emma L. Slade. Sharing of Sponsored Advertisements on Social Media: A Uses and Gratifications Perspective. The Author(s) 2018, cited in Boland, H. (2017). Facebook revenue soars as mobile advertising sales boom.

18. Karolien Poels \&Siegfried Dewitte. Getting a Line on Print Ads: Pleasure and Arousal Reactions Reveal an Implicit Advertising Mechanism. Pages 63-74 | Published online: 04 Mar 2013

19. Virda Setyani, Yu-Qian Zhu, Achmad Nizar Hidayanto, Puspa Indahati Sandhyaduhita, Bo Hsiao. Exploring the psychological mechanisms from personalized advertisements to urge to buy 
impulsively on social media. International Journal of Information Management. Volume 48, October 2019, Pages 96-107

20. https://www.sohu.com/a/318924804_120050174 